\title{
Polarity Determination in GaN Nanowires by Electron Backscatter Diffraction
}

\author{
G. Naresh-Kumar ${ }^{1}$, C. Trager-Cowan ${ }^{1}$ and A. Winkelmann ${ }^{2}$ \\ ${ }^{1}$ Department of Physics, SUPA, University of Strathclyde, Glasgow, G4 0NG, UK \\ ${ }^{2}$ Laser Zentrum Hannover e.V., Hollerithallee 8, 30419 Hannover, Germany \\ * Corresponding author: naresh.gunasekar@strath.ac.uk
}

The polarity of a crystal surface plays a crucial role in determining many of its physical properties and can also influence the nucleation of subsequent growth on those surfaces. For example in the growth of GaN, N-polar (000-1) is less stable and decomposes more easily than Ga-polar (0001). The effect of crystal polarity in wurtzite semiconductors (in particular III-nitrides) is highly influential in determining the electrical and optical properties of these material systems. Onedimensional structures such as $\mathrm{GaN}$ nanowires are highly sought after as building blocks for applications in nano-photonics and electronics for its defect free, large surface area and quantum confinement effects. The lack of inversion symmetry along the c-axis of the wurtzite GaN structure causes different polarization induced electric fields for $\mathrm{N}$ and Ga polar surfaces. N-polar surfaces offer a great advantage for high electron mobility transistors providing a strong back barrier layer, low resistivity Ohmic contacts and improved capability for large scale device processing. Recently there has been immense interest in determining polarity in semiconductor based nanowires in order to better understand their polarity dependent growth mechanisms.

A range of methods have been used to determine the polarity in GaN nanowires and nanostructures. A number of these are based on transmission electron microscopy (TEM), including convergent beam electron diffraction; and annular bright field atomic imaging in the aberration corrected scanning TEM. Whilst excellent for characterising individual nanowires, they require complex sample preparation and usually fail to sample a statistically significant number of nanowires. There are benefits in identifying a non-destructive, large-area, method for nanowire polarity determination using scanning electron microscopy (SEM). This is the subject of this work-which employs electron backscatter diffraction (EBSD) imaging in a field emission scanning electron microscope to determine the crystal polarity.

In the present work, we exploit the dynamical electron diffraction effects observed in the EBSD patterns. For example, the in-equivalence of the Bragg reflections on either side of certain Kikuchi bands leads to an asymmetric band profile, with the intensity maximum slightly shifted from the centre of these Kikuchi bands. We have used this asymmetric intensity distribution to determine the crystal polarity (see Fig. 1a-f). The EBSD patterns from the GaN nanowires is analysed using the pattern matching method described in references [1-3]. In summary, based on a starting orientation identified by the EBSD manufacturer software, we first look for an optimized crystal orientation by maximizing the normalized cross-correlation coefficient $r$ [3] between the experimental pattern and simulated pattern. As the manufacturer orientations do not account for non-centrosymmetric structures, we then explicitly test for a possible better fit of the inverted GaN crystal structure. This is equivalent to the effect of a two-fold rotation of the simulated data perpendicular to the polar c-axis direction. This makes it possible to absolutely determine the positive direction of the c-axis up the nanowire, i.e. whether [0001] ("Ga polarity") or [000-1] ("N polarity"). To quantify nanowires with different polarity, we have also acquired an EBSD map covering a range of nanowires of various dimensions (see Fig. 1g-h). Most of the nanowires are observed to be predominantly Ga-polar; however there are a number of nanowires with both polarities present. The approach described in our work can be adopted for characterising a wide range of non-centrosymmetric nanoscale material systems ranging from quantum dots and nanowires. 
References:

1. Winkelmann, A. and Nolze, G. Applied Physics Letters. 106, (2015) p. 072101-1.

2. Winkelmann, A., Nolze, G., Himmerlich, M., Lebedev, V. and Reichmann, A. Proceedings of the 6th International Conference on Recrystallization and Grain Growth, USA, (2016) p. 281.

3. Naresh-Kumar, G., Vilalta-Clemente, A., Jussila, H., Winkelmann, A., Nolze, G., Vespucci, S., Nagarajan, S., Wilkinson A. J., Trager-Cowan, C., Scientific Reports. 7, (2017). p. 10916

4. The authors acknowledge Prof. Tao Wang's group at the University of Sheffield for providing the GaN nanowire sample and support from the EPSRC grant; manufacturing of nano - engineered III-nitride semiconductors (EP/M015181/1).
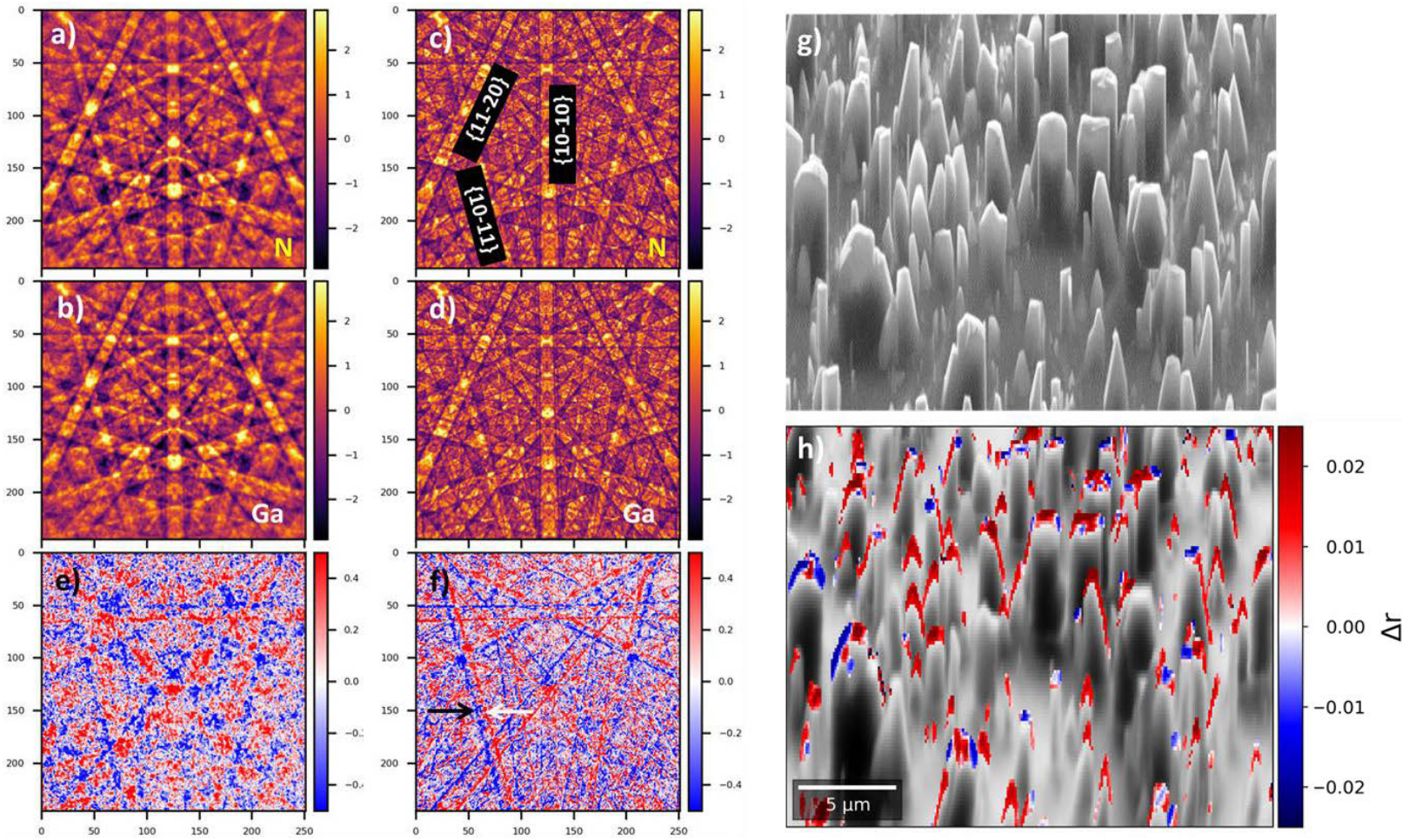

Figure 1. (a) Experimental EBSD pattern from the N polar region of the nanowire; (b) experimental EBSD pattern from the Ga polar region of the nanowire; $(c-d)$ respective simulated patterns for (a and b); (e) normalised intensity difference image of experimental EBSD patterns (from a and b); and (f) normalised intensity difference image of simulated EBSD patterns (from $\mathrm{c}$ and $\mathrm{d}$ ). The white arrow points to $\{10-11\}$ and the black arrow points to $\{10-1-1\}$; (g) Backscatter electron image acquired using solid state detector and (h) Backscatter electron intensity image derived from the raw EBSD patterns underlay on the corresponding cross-correlation difference map showing Ga oriented wires in red and $\mathrm{N}$ oriented wires in blue. Some nanowires also show both the crystal polarities. 